\title{
A Comparison of Coherence-Based Beamforming Techniques in High Frame-Rate Ultrasound Imaging with Multi-Line Transmission
}

\author{
Giulia Matrone*, Member, IEEE, Alessandro Ramalli, Senior Member, IEEE, Jan d'Hooge, Member, \\ IEEE, Piero Tortoli, Fellow, IEEE, and Giovanni Magenes, Senior Member, IEEE
}

\begin{abstract}
One of the current challenges in ultrasound imaging is achieving higher frame rates, particularly in cardiac applications, where tracking the heart motion and other rapid events can provide potential valuable diagnostic information. The main drawback of ultrasound high frame rate strategies is that usually they partly sacrifice image quality in order to speed-up the acquisition time. In particular, multi-line transmission (MLT), which consists in transmitting multiple ultrasound beams simultaneously in different directions, has been proven able to improve frame rates in echocardiography, unfortunately generating artifacts due to inter-beam crosstalk interferences.

This work investigates the possibility to effectively suppress crosstalk artifacts in MLT, while improving image quality by applying beamforming techniques based on backscattered signals spatial coherence. Several coherence-based algorithms (i.e. ShortLag Filtered Delay Multiply and Sum beamforming, Coherence and Generalized Coherence Factor, Phase and Sign Coherence, non-linear beamforming with $p$-root compression) are implemented and compared, and their performance trends are evaluated when varying their design parameters. Indeed, experimental results of phantom and in vivo cardiac acquisitions demonstrate that this class of algorithms can provide significant benefits as compared to Delay and Sum, well suppressing artifacts (up to $48.5 \mathrm{~dB}$ lower crosstalk) and increasing image resolution (by up to $46.3 \%$ ) and contrast (by up to $30 \mathrm{~dB}$ in terms of contrast ratio, and $12.6 \%$ for generalized contrast-to-noise ratio) at the same time.
\end{abstract}

Index Terms-Beamforming, Coherence Factor, Filtered-Delay Multiply and Sum Beamforming, High frame rate, Multi-Line Transmission, Phase Coherence, Sign Coherence, Spatial Coherence, Ultrasound imaging

\section{INTRODUCTION}

$\mathrm{E}$ CHOCARDIOGRAPHY is nowadays one of the routine imaging modalities for the non-invasive inspection of the

Manuscript received June 14, 2019. Asterisk indicates corresponding author. This work was partially supported by the University of Pavia under the Blue Sky Research project MULTIWAVE. A. Ramalli was supported by the European Union's Horizon 2020 research and innovation programme under the Marie Skłodowska-Curie grant agreement No 786027 (ACOUSTIC project).

*G. Matrone is with the Dipartimento di Ingegneria Industriale e dell'Informazione and with the Center for Health Technologies, Università degli Studi di Pavia, 27100 Pavia, Italy (e-mail: giulia.matrone@unipv.it). heart and the diagnosis of cardiovascular pathologies, thanks to its good spatial and temporal resolution, relative low-cost, and lack of ionizing radiation exposition. Typically, frame rates for 2D image acquisitions in clinical scanners are lower than 100 $\mathrm{Hz}$ [1], being limited by the time required to sequentially transmit and receive a series of focused beams, scanning the whole region of interest. However, at those frame rates, rapid heart motions or cardiac events would be missed; therefore, gaining higher frame rates represents one of the main challenges in echocardiography. Cardiac high-frame rate (HFR) imaging could indeed provide potential benefits on intra-frame motion artifacts and in the diagnosis and follow up of cardiovascular diseases [2]-[8].

Several HFR techniques have been proposed recently, which operate in both transmission (TX) and reception (RX) to speedup the acquisition time of each frame [9]. These techniques are based on the transmission of either defocused beams, such as plane and diverging waves [10], [11], or multiple focused beams as in multi-line transmission (MLT) [12], [13]. In RX, they implement multi-line acquisition (MLA) or parallel beamforming, to form beams along more than one scan direction simultaneously [14], [15].

Although these methods allow achieving very high frame rates (e.g., with plane waves, images can be acquired even at about $10 \mathrm{kHz}$ ), at the same time they sacrifice image quality, either in terms of contrast, resolution or both. In plane and diverging wave imaging, the lack of focusing degrades image quality [16], showing coarse resolution and edge-wave related artifacts [16], [17]. Similarly to plane/diverging wave imaging, also MLT implies a trade-off between image quality and frame rate improvement, since the so called crosstalk artifacts appear in both the axial and lateral directions due to inter-beam interference [18], decreasing image contrast. Several possible

A. Ramalli and J. D'Hooge are with the Laboratory on Cardiovascular Imaging and Dynamics, Dept. of Cardiovascular Sciences, KU Leuven, 3000 Leuven, Belgium.

P. Tortoli is with the Dipartimento di Ingegneria dell'Informazione, Università degli Studi di Firenze, 50121 Florence, Italy.

G. Magenes is with the Dipartimento di Ingegneria Industriale e dell'Informazione and with the Center for Health Technologies, Università degli Studi di Pavia, 27100 Pavia, Italy. 
solutions have been proposed to address these problems for the abovementioned HFR techniques, including the application of spatial compounding [10], [16] or of properly designed apodization weights [18]-[20], or beamforming strategies more efficient than standard Delay and Sum (DAS) [17], [21]-[23], including coherent and adaptive weighting [24]-[26]. However, the advantage of MLT imaging is that it has lower requirements in terms of beamforming speed than other HFR methods providing comparable image quality and similar frame rates (in the order of hundreds of $\mathrm{Hz}$ ), as discussed in [1].

In another study [27], we showed that MLT has a detrimental impact on the spatial coherence of backscattered echoes, which is heavily influenced by the number and angular spacing of simultaneous TX beams. Therefore, the aim of this paper is:

1. to introduce coherence-based beamforming methods in MLT imaging;

2. to compare the imaging performance of such coherence-based beamformers, by analyzing their behavior with either single focused beams (single line transmission, SLT) and MLT.

This study includes beamforming with Coherence Factor (CF) and Generalized CF (GCF) weighting [28], Filtered Delay Multiply and Sum (FDMAS) [29] and Short-Lag-FDMAS (SLFDMAS) [27] algorithms, non-linear beamforming based on $p$ root compression ( $p$-DAS) [30], and Phase and Sign Coherence (PC, SC) beamformers [31].

Section II introduces the MLT technique and the steps involved by each of the analyzed beamformers; the experimental setup and measured outcome parameters are also illustrated. Section III presents the results obtained in phantom experiments and in in vivo cardiac imaging. The performance of the abovementioned methods is further analyzed and discussed in Section IV, where some conclusions are provided too.

\section{BEAMForming TECHNIQUES IN TRANSMisSION AND RECEPTION}

\section{A. Multi-Line Transmission}

MLT achieves high frame rates by transmitting at the same time $N_{T X}$ beams along different focusing directions, and then beamforming in parallel the signals backscattered along the abovementioned directions. Specifically, the frame rate is increased $N_{T X}$ times compared to the standard SLT scan sequences.

In order to do so, the excitation signal is calculated as the sum of the excitation pulses that would be used in conventional SLT to focus along each one of these directions. The $N_{T X}$ beams are thus transmitted and then moved by an angular step; by repeating this process, a full angular sector $\theta$ is covered. If this sector consists of $N_{\text {LINES }}$ scan lines, then the step angle is $\theta_{\text {STEP }}=\theta / N_{\text {LINES }}$, and the TX beams are separated by an angle $\theta_{T X}=\theta / N_{T X}[18]$.

As it often occurs with HFR techniques, improvements in the acquisition time come at the expense of image quality. In this case, the so-called crosstalk artifacts arise in MLT images, since interferences occur among the simultaneously transmitted beams. In particular, TX crosstalk is defined as the result of interferences between sidelobes of the TX beam and the main lobe of the RX beam, while RX crosstalk is due to interferences among the TX beam main lobe and RX beam side lobes [18]. In the point spread function (PSF) they appear as side lobes along the range and cross directions, respectively.

\section{B. Coherence Factor}

CF was first introduced in [32], [33] as a parameter able to measure the quality of RX focusing; later, it was proposed as a weighting factor to be applied to the beamformed signals [28]. The CF is formally defined as the ratio of the coherent and incoherent sum of radiofrequency (RF) signals $s_{n}(t)$ received by $N$ active elements, after the application of RX focusing delays:

$$
C F(t)=\frac{\left|\sum_{n=1}^{N} s_{n}(t)\right|^{2}}{N \sum_{n=1}^{N} s_{n}^{2}(t)} .
$$

$\mathrm{CF}$ approaches 1 when the coherence of signals is high, i.e. when the focusing quality is good; otherwise, CF assumes lower values, down to 0 . If used as a weighting factor, each beamformed line of the CF-weighted final image is obtained as:

$$
y_{C F}(t)=C F(t) y(t)
$$

where $y(t)$ is the DAS beamformer output:

$$
y(t)=\sum_{n=1}^{N} s_{n}(t) .
$$

\section{Generalized Coherence Factor}

GCF [28] is a generalization of the CF. It is based on the computation of the spectrum $S_{t}(k)$ of $s_{n}(t)$. Then, GCF is defined as the ratio between the energy at frequencies lower than a cutoff frequency $M_{0}$, and the total energy:

$$
G C F(t)=\frac{\sum_{k=0}^{M_{0}}\left|S_{t}(k)\right|^{2}}{\sum_{k=0}^{N-1}\left|S_{t}(k)\right|^{2}}
$$

where $k$ represents the spatial frequency index. In practice, this means computing the ratio between the energy received from directions around the TX beam and the energy received from all directions. Besides, $\mathrm{GCF}$ reduces to $\mathrm{CF}$ when $M_{0}=0$, i.e. only the DC spectral component is considered.

$M_{0}$ represents a spatial frequency, thus in theory its value is in the range [0;N/2-1]. In [28] it was shown that sidelobe suppression decreases as $M_{0}$ increases; on the other hand, $M_{0}>0$ should be selected in order to avoid beam-splitting artifacts due to phase aberrations and artificial black holes in speckle. However, since a too high $M_{0}$ reduces the effectiveness of this technique, values in the range 1-3 should be generally preferred for imaging of diffuse scatterers [28].

The GCF-weighted image is obtained as: 


$$
y_{G C F}(t)=G C F(t) y(t) .
$$

\section{Phase Coherence and Sign Coherence}

$\mathrm{PC}$ was proposed in [31] to evaluate the coherence of phases of signals after RX dynamic focusing, which should be higher where the beam is better focused, and thus where spatial coherence increases. The PC factor (PCF) is computed as:

$$
P C F(t)=\max \left\{0,1-\frac{\gamma}{\sigma_{0}} s f(t)\right\}
$$

where $\sigma_{0}=\pi / 3^{1 / 2}$ is the standard deviation of a uniform distribution in $[-\pi ; \pi]$ and $\gamma$ is a user-defined parameter in the range $[0 ; 1] . s f(t)$ is defined as:

$$
s f(t)=\min \left\{\sigma\left(\phi_{n}(t)\right), \sigma\left(\phi_{n}^{A}(t)\right)\right\}
$$

where $\sigma\left(\phi_{n}(t)\right)$ is the standard deviation (in the RX aperture spatial domain) of the instantaneous phases $\phi_{n}(t)$ of received (and delayed) signals $s_{n}(t)$ at discrete time instant $t$, and $\phi^{A}{ }_{n}(t)$ is equal to $\phi_{n}(t)+\pi$ when $\phi_{n}(t)<0$, or to $\phi_{n}(t)-\pi$ when $\phi_{n}(t)>0$.

SC [31] instead considers the distribution of signs of received signals, therefore using a single bit to represent their instantaneous phase; i.e., for each time instant $t, b_{n}(t)=-1$ if $s_{n}(t)<0$, otherwise $b_{n}(t)=+1$. The SC Factor (SCF) is thus defined as:

$$
S C F(t)=\left|1-\sigma\left(b_{n}(t)\right)\right|^{q}
$$

where $\sigma\left(b_{n}(t)\right)$ is the standard deviation of the signal sample signs $b_{n}(t)$ (in the RX aperture spatial domain), and $q \geq 0$ is again a user-defined parameter for SCF sensitivity tuning.

PCF and SCF can be then used to weight the DAS beamformed output $y(t)$ as follows:

$$
\begin{aligned}
& y_{P C F}(t)=P C F(t) y(t), \\
& y_{S C F}(t)=S C F(t) y(t) .
\end{aligned}
$$

In [31] it was shown that, by increasing $\gamma$ and $q$ up to 1 , for PCF and SCF respectively, higher imaging performance in terms of side-lobe suppression and narrowing of the main lobe (i.e. contrast and resolution) could be obtained; on the opposite, $\gamma$ and $q$ values equal to 0 made these algorithms behave as DAS.

\section{E. Short-Lag Filtered Delay Multiply and Sum Beamforming}

FDMAS beamforming, which is based on the computation of the RX aperture spatial autocorrelation function, was proposed in [29]. It was then generalized in [27] to consider only a certain maximum lag in the computation of the autocorrelation $(M L)$, leading to a different formulation known as SL-FDMAS:

$$
y_{S L-D M A S}(t)=\sum_{L=1}^{M L} \sum_{n=1}^{N-L} \operatorname{sign}\left(s_{n}(t) s_{n+L}(t)\right) \cdot \sqrt{\left|s_{n}(t) s_{n+L}(t)\right|}
$$

where $s_{n}(t)$ are the received RF signals after the application of $\mathrm{RX}$ focusing delays. As the cross-multiplication operation alters the output signal frequency content, generating both a second harmonic component at $2 f_{0}$ (where $f_{0}$ is the TX central frequency) and a baseband component, $y_{S L-F D M A S}(t)$ is subsequently obtained by band-pass (BP) filtering $y_{S L-D M A S}(t)$ on the second harmonics only, in order to attenuate as much as possible other frequency components which originate from such non-linear operations. SL-FDMAS equals FDMAS when $M L$ is set to $N-1$.

By tuning $M L$, a trade-off between resolution and contrast exists, as demonstrated in [27]: in general, lower $M L$ values imply higher lateral resolution but also higher side-lobes, i.e. lower contrast, while the opposite effect is achieved by increasing $M L$.

\section{F. Non-Linear Beamforming with p-root Compression}

Starting from the FDMAS expression, in [30], [34] a nonlinear beamformer based on $p$-th root compression ( $p$-DAS) was proposed by generalizing this formalism through the use of a $p$-th root and $p$-th power as follows:

$$
y_{P-D A S}(t)=\operatorname{sign}\left\{\sum_{n=1}^{N} \operatorname{sign}\left(s_{n}(t)\right) \sqrt[p]{s_{n}(t)}\right\} \cdot\left|\sum_{n=1}^{N} \operatorname{sign}\left(s_{n}(t)\right) \sqrt[p]{s_{n}(t)}\right|^{p} .
$$

Also in this case, the non-linear $p$-th root and $p$-th power operations distort the frequency content of the output signal, in which artificial harmonics at multiples of the TX central frequency appear. Thus, in the end $y_{p-D A S}(t)$ has to be BP-filtered around the fundamental frequency component. Like FDMAS, $p$-DAS is a coherence-based beamformer, where the $p$ parameter can be used to tune the contrast/resolution performance of the algorithm on the final image. Increasing $p$ generally improves resolution and contrast, but degrades speckle uniformity [30].

\section{G. Ultrasound Imaging Setup}

Experimental acquisitions were performed by means of the 64-channel ULA-OP research scanner [35], [36] connected to a 128 -element phased array probe (model PA230, Esaote S.p.A., Florence, Italy) with a $170 \mu \mathrm{m}$ pitch. Only the 64 odd elements of the probe were used in order to maximize the aperture width, so the actual pitch was $340 \mu \mathrm{m}$. The central frequency was 2 $\mathrm{MHz}$, and a 2-cycle Hanning-weighted sinusoidal burst was used as the excitation signal. Its amplitude was $25 \mathrm{~V}_{\mathrm{pp}}$ in the SLT case, while in MLT it was reduced proportionally to the number of TX beams, i.e. $25 / N_{T X} \mathrm{~V}_{\mathrm{pp}}$, since $25 \mathrm{Vpp}$ was the maximum output amplitude of the linear transmitter onboard the ULA-OP system. Moreover, as shown in [37], with such amplitude limitation MLT's safety is guaranteed without risks of any thermal or mechanical effect. The TX focus was set at $70 \mathrm{~mm}$ depth. Pre-beamforming signals were collected by the probe, sampled at $50 \mathrm{MHz}$ and sent to the computer via USB for beamforming and image analysis. Acquisitions were carried out using two different phantoms. The first one consisted of four vertically-aligned nylon wires $(100 \mu \mathrm{m}$ diameter) immersed in water at increasing depths, while the second one was a commercial tissue-mimicking phantom (model 040GSE, 
CIRS Inc, Norfolk, VA, USA). Also in vivo cardiac imaging experiments were performed on a young healthy volunteer. This study was approved by the local ethics committee, and informed consent was received from the participant.

Data from a $90^{\circ}$-wide image sector corresponding to 192 scan lines were acquired. After beamforming, the obtained RF image lines were demodulated using the Hilbert transform, normalized and logarithmically compressed to obtain the final B-mode image. SLT and MLT with 4, 6 and 8 TX beams (i.e. 4-, 6-, 8-MLT) were implemented. Tukey apodization was applied in TX in all cases, to reduce TX crosstalk, as proposed in [18], while the following techniques for RX crosstalk suppression were compared: DAS with Tukey RX apodization (DAS-T), SL-FDMAS with $M L$ equal to 10 or 32, and standard FDMAS $(M L=63) ; p$-DAS with $p=3$ and 4 ; CF-weighted DAS; PCF and SCF beamforming with $\gamma$ and $q$ equal to 0.2, 0.4, 0.6, $0.8,1$, respectively; GCF with $M_{0}=1,2,3,4,5$. For SL-FDMAS and $p$-DAS signals, the BP filter frequency boundaries were set to 2-6 MHz and 1-3 MHz, respectively.

\section{H. Image Quality Metrics}

Imaging performance was evaluated in terms of lateral resolution (LR) at $-6 \mathrm{~dB}$, considering the wire in water at 75 $\mathrm{mm}$ depth (i.e. nearest to the TX focus), of contrast ratio (CR) and generalized contrast-to-noise ratio (GCNR, [38]):

$$
\begin{gathered}
C R=20 \log _{10}\left(\frac{\mu_{c y s t}}{\mu_{b c k}}\right) \\
G C N R=1-\int \min \left\{p_{c y s t}(\hat{y}), p_{b c k}(\hat{y})\right\} d \hat{y}
\end{gathered}
$$

where $\mu_{c y s t}$ and $\mu_{b c k}$ represent the mean of envelope-detected signal values (before log-compression) inside the cyst and in the background, respectively, while $p_{c y s t}$ and $p_{b c k}$ are the probability density functions of the enveloped signal $\hat{y}$ inside and outside the cyst, respectively. The GCNR in fact provides an analysis of contrast-to-noise ratio (CNR) that takes into account the probability density function of image values inside and outside the cyst target, so as to be resistant to dynamic range alterations. We decided to consider such metrics since nonlinear and/or adaptive beamforming algorithms could change the beamformed signals dynamic range [39], thus comparisons in terms of standard CNR could be altered as a consequence. In this work, two circular areas ( $5 \mathrm{~mm}$ diameter) at the same depth, inside and outside the phantom anechoic cyst at $\sim 70 \mathrm{~mm}$, were considered to compute the abovementioned parameters (see yellow circles in Fig. 2). Besides, RX crosstalk (XT) was evaluated by computing the ratio between the peak amplitude of the Point Spread Function (PSF) (i.e. the wire in water) at 75 $\mathrm{mm}$ depth and the highest peak of artifacts due to crosstalk.

\section{RESUlts}

\section{A. Phantom Images}

Images of the nylon wires immersed in water are presented in Fig. 1, while Fig. 2 shows CIRS phantom images; in both cases only 4-MLT was considered, to permit a qualitative comparison between images obtained with different beamformers. In Fig. 3 the measured parameters (LR, XT, CR and GCNR) are reported, again in the 4-MLT case. A full representation of all the obtained outcome metrics is instead provided by the bar plots in Fig. 4, where bars represent relative values obtained with the different beamformers and SLT/MLT, with respect to DAS-T ones. Normalization values, i.e. the measurements obtained with DAS-T beamforming, are listed in Table I as a reference.

By looking at Fig. 1, it can be immediately noticed that an improvement in lateral resolution occurs when coherence-based
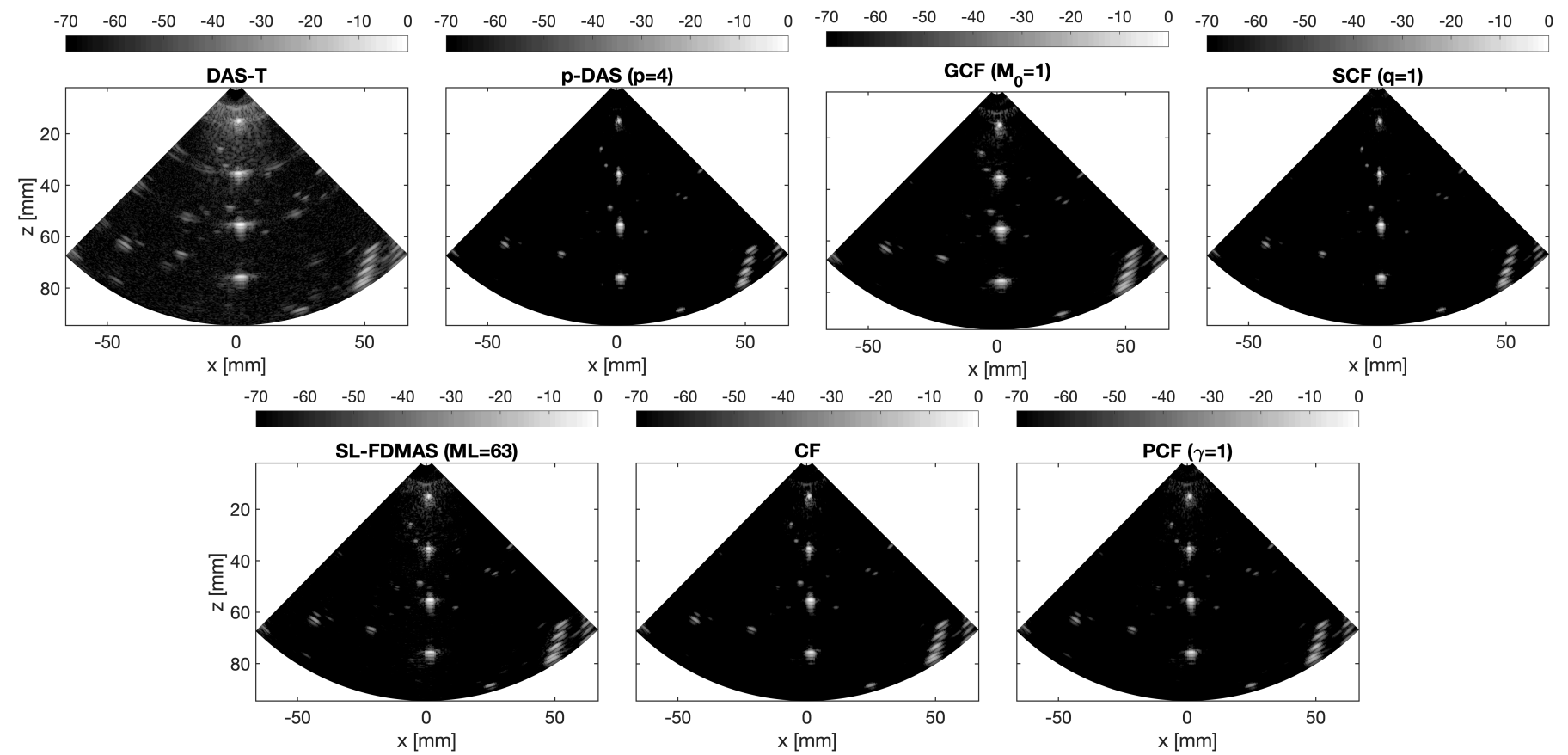

Fig. 1. Images of the nylon wires in water, acquired with 4-MLT and different RX beamformers, displayed over a log-scale with a $70 \mathrm{~dB}$ dynamic range: DAS with Tukey RX apodization, $p$-DAS with $p=4$, GCF with $M_{0}=1$, SCF with $q=1$, SL-FDMAS with $M L=63$ (i.e. FDMAS), CF, and PCF with $\gamma=1$. 




Fig. 2. Images of the CIRS phantom, acquired with 4-MLT and different RX beamformers, displayed over a log-scale with different dynamic ranges: DAS with Tukey RX apodization, $p$-DAS with $p=4$, GCF with $M_{0}=1$, SCF with $q=1$, SL-FDMAS with $M L=63$ (i.e. FDMAS), CF, and PCF with $\gamma=1$. Yellow circles on the DAS-T image represent areas used to evaluate performance metrics.

beamforming methods are used, as nylon wires at all depths look narrower than DAS-T ones. With DAS-T, a LR of about $2.6 \mathrm{~mm}$ is achieved with both SLT and MLTs, while with coherence-based beamformers LR ranges from $1.4 \mathrm{~mm}$ with SL-FDMAS $(M L=10)$ to $2.2 \mathrm{~mm}$ with GCF (Fig. 3), i.e. improvements of $\sim 46 \%$ and $\sim 14 \%$, respectively, as compared to DAS-T (Fig. 4). Very good values (about 1.5-1.7 mm, $40 \%$ $33 \%$ better than DAS-T) are achieved also with $p$-DAS, PCF and SCF with high $\gamma$ and $q$ values, respectively. Almost the same results are obtained again for all MLTs. In general, decreasing $M L$ or $M_{0}$ and increasing $p, \gamma$ or $q$ makes SLFDMAS, GCF, $p$-DAS, PCF and SCF achieve a better resolution, respectively (Fig. 4). LR with CF is better $(1.9 \mathrm{~mm}$ vs. $2.2 \mathrm{~mm}$ ) than with GCF (Fig. 3-4).

All coherence-based beamformers achieve better performance than DAS-T in terms of RX crosstalk rejection too (Fig. 4), with PCF at low $\gamma$ values the only exception (e.g., a 4.4 $\mathrm{dB}$ higher XT is obtained in 6-MLT with $\gamma=0.2)$. Actually, also GCF with $M_{0}=5$ is outperformed by DAS-T in 8-MLT (Fig. 4), but only by $0.5 \mathrm{~dB}$. The abovementioned improvement can be observed by looking at Fig. 1, where only the DAS-T image shows visible RX crosstalk peaks at the left and right sides of each nylon wire. For example, XT with DAS-T goes from -42.8 $\mathrm{dB}$ in 4-MLT to $-34 \mathrm{~dB}$ in 8-MLT (Table I), while it reaches values down to $-91.3 \mathrm{~dB}$ and $-81.5 \mathrm{~dB}$ with $p$-DAS $(p=4)$ in 4MLT and 8-MLT, respectively (Fig. 4). Moreover, an improved XT-rejection can be observed when increasing $M L, p, \gamma$, and $q$ for SL-FDMAS, $p$-DAS, PCF and SCF, respectively. For $\mathrm{CF} / \mathrm{GCF}$ instead XT is better suppressed when decreasing $M_{0}$ from 5 to 0 (Fig. 4). If we consider 4-MLT (Fig. 3), for example, in the best case we have a stronger XT rejection of $-19.2 \mathrm{~dB}$ for FDMAS, $-48.5 \mathrm{~dB}$ for $p$-DAS ( $p=4),-36.6 \mathrm{~dB}$ for $\mathrm{CF},-29.1 \mathrm{~dB}$ for GCF $\left(M_{0}=1\right),-16.6 \mathrm{~dB}$ for PCF $(\gamma=1)$ and $-31 \mathrm{~dB}$ for SCF $(q=1)$, with respect to DAS-T.

Two further plots, showing the lateral and axial sections of the wire at $75 \mathrm{~mm}$ depth have been provided as supplementary material (Figure 1S.tiff). In these plots, sidelobes corresponding to RX and TX crosstalk artifacts have been further highlighted by colored arrows.

Images of the tissue mimicking phantom (Fig. 2), are displayed over different dynamic ranges, chosen in order to obtain a histogram of pixel gray-scale levels almost equal to that of DAS-T. This procedure was implemented so as to provide a better visibility to images, in particular to those obtained with coherence-based beamformers, which, due to the improved contrast resolution, usually look darker and with more black areas in the background [39]. Even if in this way almost the same mean gray level is obtained for all images, Fig. 2 shows that coherence-based beamformers are still able to bring improvements as compared to DAS-T, with images looking better resolved (for example, refer to the 3 wires aligned along the horizontal axis at $z=40 \mathrm{~mm}$ ) and with a higher contrast. This is confirmed by CR values in Fig. 3-4, showing that all coherence-based beamformers achieve a higher contrast than DAS-T. Up to $13 \mathrm{~dB}$ improvement is generally obtained by FDMAS with respect to DAS-T, which decreases if considering only short lags (Fig. 4). $p$-DAS reaches a CR up to $30 \mathrm{~dB}$ higher (in absolute value) than DAS-T in 4-MLT; a $\sim 20$ $\mathrm{dB}$ improvement is also achieved by $\mathrm{CF}$ and GCF with $M_{0}=1$, while PCF with $\gamma=1$ and SCF with $q=0.8$ gain about $15 \mathrm{~dB}$ and $18 \mathrm{~dB}$, respectively (Fig. 4). As observed for XT suppression, also CR improvement generally increases with $M L, p, \gamma$ and $q$ for SL- FDMAS, $p$-DAS, PCF and SCF, respectively (Fig. 4). For $\mathrm{CF} / \mathrm{GCF}$ instead, increasing $M_{0}$ leads to a decrease of $\mathrm{CR}$ improvement. 
Finally, in terms of GCNR, in SLT mode GCF is the only algorithm providing a slight improvement with respect to DAS$\mathrm{T}$ (Fig. 4). Whereas, some significant differences are noticeable in the MLT cases. Almost all coherence-based algorithms in fact show a higher GCNR than DAS-T with MLT, in particular for increasing $N_{T X}$, except for SL-FDMAS with very short lags $(M L=10)$ and SCF with $q=0.8-1$ (Fig. 4). GCNR improves for decreasing $p$ and $q$ values in $p$-DAS and SCF, respectively, or for increasing $M L$ values in SL-FDMAS. For PCF instead we can see that GCNR remains almost constant up to $\gamma=0.8$, while for $\gamma=1$ it slightly decreases (Fig. 3). Finally, GCNR for GCF is almost constant with $M_{0}$, but always higher than for DAS-T and also slightly higher than for $\mathrm{CF}$, which reaches values similar to those of $p$-DAS with $p=4$ (Fig. 3).

In 4- and 6-MLT the best GCNR is achieved by $p$-DAS with $p=3$ ( 0.92 vs. 0.87 with DAS-T in 4-MLT, and 0.82 vs. 0.73 in 6-MLT), while in SLT and 8-MLT it is achieved by GCF with $M_{0}=4$ and $M_{0}=2$, respectively ( 0.97 vs. 0.96 with DAS-T in SLT and 0.79 vs. 0.63 with DAS-T in 8 -MLT). In terms of percentage increment, this means that we have a $5.2 \%$ and $12.4 \%$ improvement with $p$-DAS $(p=3)$ in 4- and 6-MLT, and up to $25.7 \%$ improvement with $\mathrm{GCF}\left(M_{0}=2\right)$ when 8 beams are transmitted (Fig. 4). GCF, $p$-DAS and SL-FDMAS algorithms in general achieve better GCNR performance (in decreasing order) than PCF and SCF in MLT. In particular, the lowest values are achieved by SCF (4.4\% improvement at most, as compared to DAS-T, in 8-MLT with $q=0.6$ ) (Fig. 4).

A zoomed view of the cyst at about $70 \mathrm{~mm}$ depth, used for CR and GCNR evaluation, has been provided in Figure2S.tiff, included as supplementary material.

\section{B. In Vivo Cardiac Images}

Two different sets of in vivo heart images acquired with 4MLT are shown in Fig. 5 (5-chamber apical view) and Fig. 6 (4-chamber apical view). The corresponding videos are also provided as supplementary material (5Chambers $A V . m p 4$ and 4ChambersAV.mp4).

To display these images, dynamic ranges have been again adjusted on the basis of a histogram analysis, in order to match, as well as possible, the reference histogram of the DAS-T image. In spite of this, both figures show that with coherencebased beamformers an improved contrast can be obtained with respect to DAS-T. The four chambers look darker, as well as the aorta in Fig. 5, and those artifacts that affect the visibility of atria in the DAS-T image (especially inside the right atrium and outside the left one, see yellow arrows in the DAS-T panel of Fig. 5 and Fig. 6) are almost no more present in the other images, thanks to the higher crosstalk suppression. The higher contrast makes also the septum and mitral valve more visible in Fig. 5-6.

Another important benefit brought about by coherence beamformers is the improved lateral resolution, which can be noticed by looking at the speckle pattern of the myocardial walls. The only exception is probably represented by GCF images with $M_{0}=1$, in which such an improvement is limited, even if contrast looks still higher than with DAS-T.

The combination of improved contrast and resolution allows achieving, in general, sharper images, with better defined borders and more clearly visible chambers/valves. Moreover, it can be noticed that, with coherence based beamformers, even the pericardial cavity on the left ventricle external border becomes visible, particularly well in $p$-DAS and SCF images of the 5-chamber apical view (Fig. 5), but also in Fig. 6 (as pointed out by the green arrow).

In order to visualize more clearly the improvements brought about by coherence-based beamformers, we have also provided
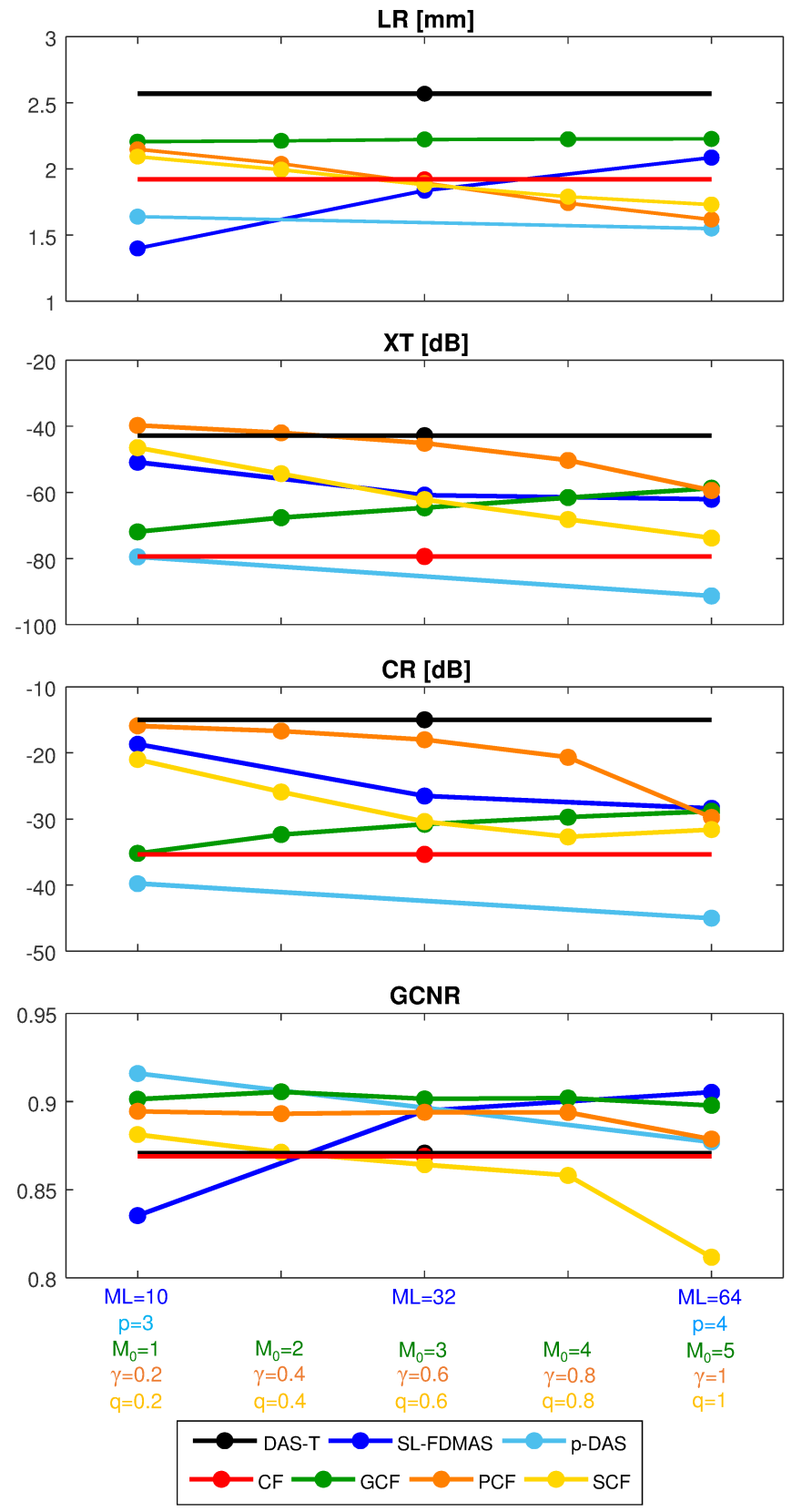

Fig. 3. Performance parameter values obtained by the different beamformers in 4-MLT.

TABLE I

MEASURED IMAge QuALITY PARAMETERS WITH DAS-T BEAMFORMING

\begin{tabular}{lcccc}
\hline \hline & LR [mm] & XT [dB] & CR [dB] & GCNR \\
\hline SLT & 2.62 & - & -22.21 & 0.96 \\
4-MLT & 2.57 & -42.82 & -14.98 & 0.87 \\
6-MLT & 2.61 & -39.20 & -11.24 & 0.73 \\
8-MLT & 2.57 & -34 & -9.20 & 0.63 \\
\hline \hline
\end{tabular}




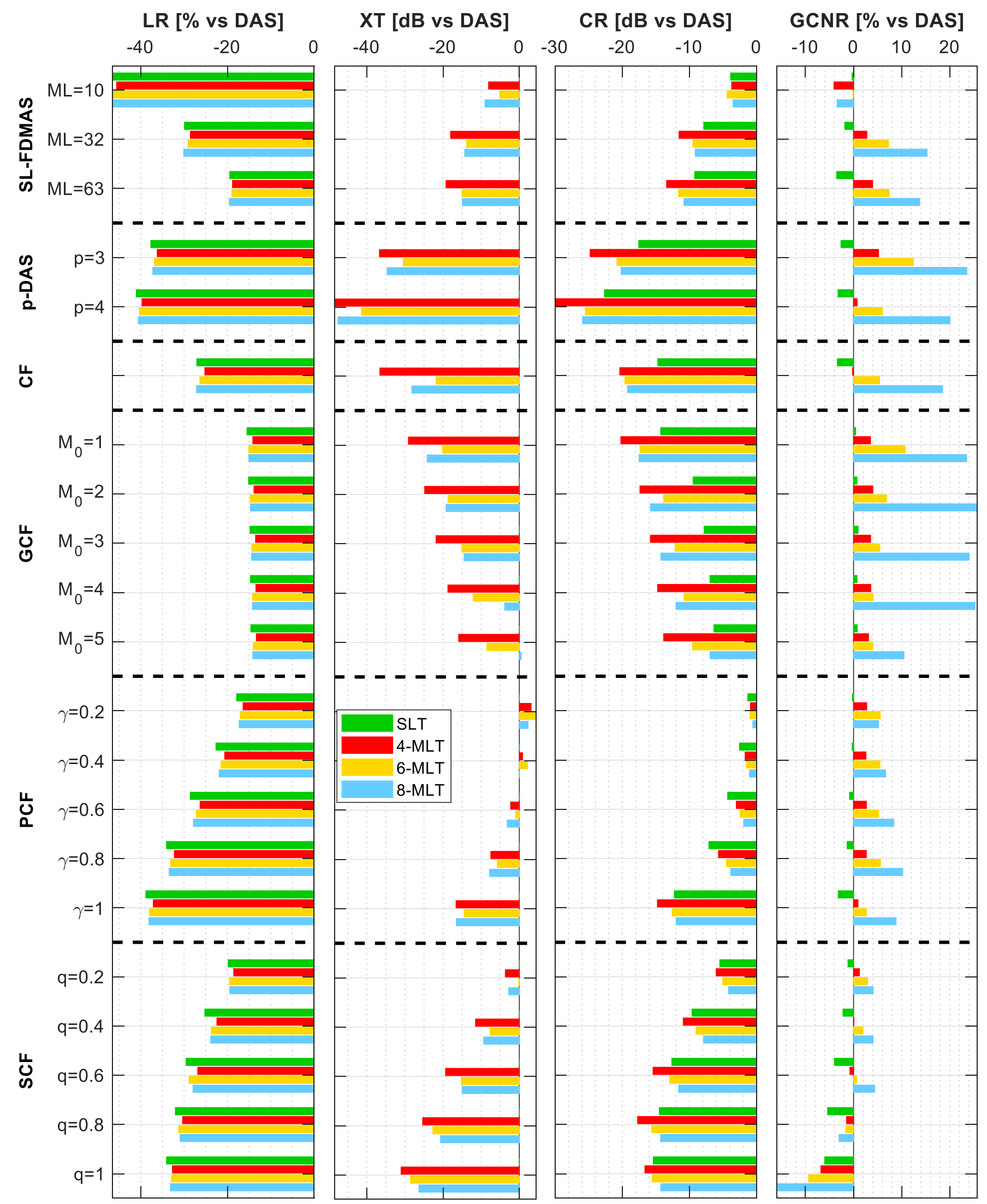

Fig. 4. Experimentally measured image quality parameters with respect to DAS-T ones (cf. Table I): (from left to right) LR, XT, CR and GCNR. 
a magnified view of the details indicated by arrows in Fig. 5 and Fig. 6; these additional images are provided as supplementary material. Figure5SA.tiff, Figure5SB.tiff, and Figure 5SC.tiff show the zoomed view of regions indicated by left/right yellow arrows and by the green arrow in Fig. 5, respectively; Figure6SA.tiff, Figure6SB.tiff and Figure6SC.tiff refer to left/right yellow and green arrows in Fig. 6, respectively.
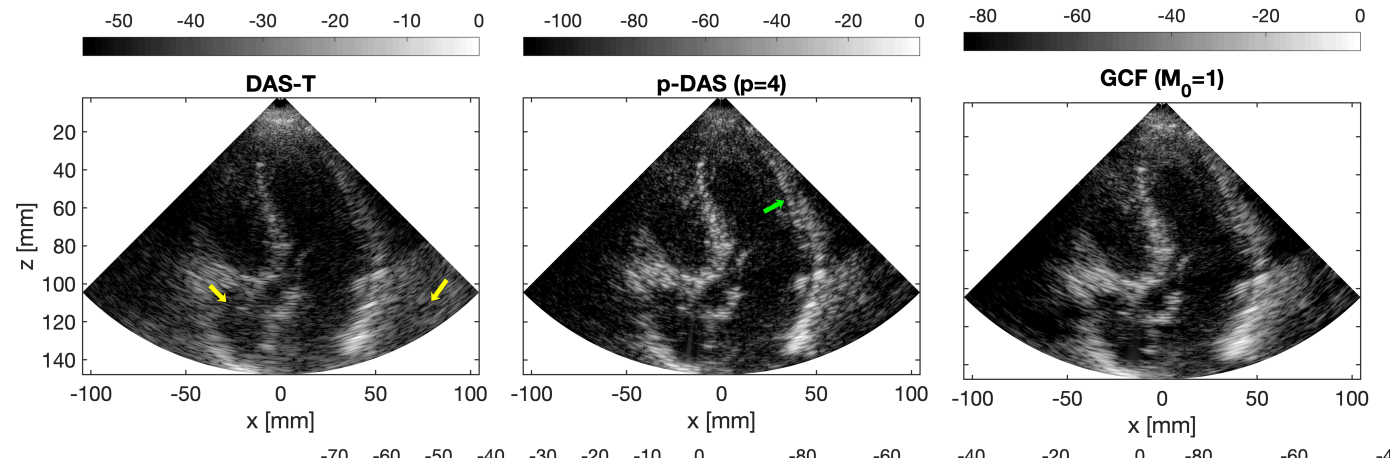

\section{DiscusSION AND CONCLUSION}

In this paper we have investigated whether beamformers exploiting the spatial coherence of backscattered signals can provide benefits in cardiac HFR imaging with MLT. Up to now, their good performance has been mainly proven in standard SLT indeed, where single focused beams are sequentially transmitted to acquire a full image. Here instead we analyze their behavior in a different scan configuration, i.e. in the presence of multiple TX beams (MLT), which are simultaneously transmitted to improve the frame rate but
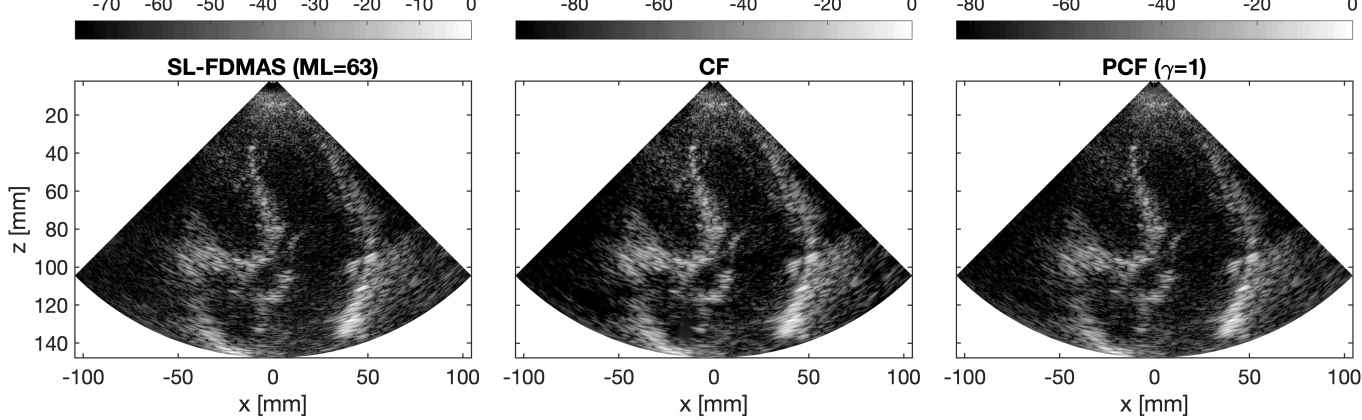

Fig. 5. 5-chamber apical views of the heart, acquired with 4-MLT and different RX beamformers, displayed over a log-scale with different dynamic ranges: DAS with Tukey RX apodization, $p$-DAS with $p=4$, GCF with $M_{0}=1$, SCF with $q=1$, SL-FDMAS with $M L=63$ (i.e. FDMAS), CF, and PCF with $\gamma=1$.


Fig. 6. 4-chamber apical views of the heart, acquired with 4-MLT and different RX beamformers, displayed over a log-scale with different dynamic ranges: DAS with Tukey RX apodization, $p$-DAS with $p=4$, GCF with $M_{0}=1$, SCF with $q=1$, SL-FDMAS with $M L=63$ (i.e. FDMAS), CF, and PCF with $\gamma=1$. 
heavily influence spatial coherence.

At the same time, this study has provided a comparative assessment of the imaging performance of six different coherence-based techniques. For each technique, contrast, resolution and inter-beam crosstalk suppression have been evaluated.

The results demonstrate that all the analyzed beamformers are able in general to provide better image quality than the reference DAS-T, lowering RX crosstalk artifacts without sacrificing resolution or contrast.

Lateral resolution, measured as the main lobe width at $-6 \mathrm{~dB}$, is not influenced by the transmission of multiple beams, since $\mathrm{RX}$ crosstalk determines side lobes separated from the main lobe. Coherence-based algorithms providing higher resolution than DAS-T in SLT behave similarly in MLT. The LR values in Table I and bar plots in Fig. 4 show that DAS-T provides the worst LR, also because of RX Tukey apodization applied to suppress crosstalk. As expected, other algorithms achieve better results, since they are known to suppress off-axis signals providing a narrow beam, thanks to the use of spatialcoherence-related weights or image formation procedures. Moreover, these beamformers show a different LR when varying their user-defined parameters. For example, LR gets worse when increasing $M L$ in SL-FDMAS images and when increasing $M_{0}$ in GCF images, while for $p$-DAS, PCF and SCF, LR improves by increasing $p, \gamma$ and $q$ parameters, respectively. The best result is that of SL-FDMAS with $M L=10$, which succeeds in obtaining a very narrow beam main lobe, using only highly coherent information in the beamforming process.

RX crosstalk suppression instead varies with the number of TX beams in MLT. For what concerns the possible dependence of crosstalk suppression on beamforming design parameters, Fig. 4 demonstrates again that for SL-FDMAS performance improves with increasing $M L$; also for $p$-DAS, PCF and SCF we have improvements when $p, \gamma$ and $q$ increase, respectively. $\mathrm{CF} / \mathrm{GCF}$ performance gets instead lower when $M_{0}$ increases. Generally, all coherence-based beamformers perform better than DAS-T; RX crosstalk is in fact reduced thanks to the lower RX beam sidelobes, which turns out also in improved contrast in MLT images and less artifacts. The only exception is PCF with $\gamma$ equal to 0.2 and 0.4 , which reaches XT peaks up to 4.4 $\mathrm{dB} \%$ higher than DAS-T ones in MLT (Fig. 4), probably because with such low $\gamma$ values RX beam sidelobes are not enough suppressed and inter-beam interferences still occur.

Crosstalk reduction has an important impact in cardiac images, where the visibility of atria is significantly improved by coherence-based beamforming, reducing all the artifacts that can be seen especially in the lower right and left regions of DAS-T images (see yellow arrows in Fig. 5 and Fig. 6).

These LR and crosstalk suppression improvements also bring about a higher CR with coherence-based beamforming (Fig. 4). In DAS-T images, both the worse resolution and all crosstalk artifacts make the background-tissue speckle look more uniform, but also cysts and heart chambers are filled up with noise (Fig. 2, 5, 6), thus contrast decreases. On the other hand, with coherence-based beamformers, the lumen of cysts and heart chambers qualitatively show a lower mean gray level; thus, in spite of the tissue speckle being darker too (due to the increased contrast resolution), CR improves.

It is known that often the CNR becomes low in high contrastresolution/dynamic-range images, because of the sharper speckle pattern with an increased variance [38], [39]. This effect can be observed in the coherence-based beamforming images, as mentioned earlier. Besides, with these algorithms, the speckle looks partially darker in MLT than in SLT, due to a decrease of spatial coherence, as shown in our previous work [27]. On the other hand, in MLT the DAS-T image speckle appears to be even more uniform due to the presence of crosstalk artifacts, higher side lobes and lower resolution. For these reasons:

i. we showed images with different dynamic ranges, in order to match as well as possible their histograms and obtain similar appearance in terms of speckle average gray level; in spite of this, coherence beamformers obtain a visibly better image quality;

ii. we evaluated the GCNR, instead of CNR, since it is immune to image dynamic-range alterations [38].

The obtained GCNR values show that all algorithms, including DAS-T, achieve almost equal results in SLT. Significant differences can be noticed as the number of simultaneous TX beams increase, in particular in 8-MLT, showing an improvement (up to 26\%) with some coherencebased beamformers (e.g. FDMAS, $p$-DAS, CF, GCF, PCF and slightly also SCF with lower $q$ ) as compared to DAS-T (Fig. 4).

This means that, in spite of possible dynamic range alterations, coherence-based beamformers are really able to improve image CR and CNR in MLT, thanks to their improved sidelobe and XT suppression capabilities, which play an important role in achieving high frame rates (as in 8-MLT) while maintaining a good image quality.

In conclusion, our study demonstrates that coherence-based

TABLE II

Algorithms Providing the Best and Worst Performance in Each CONFIGURATION

\begin{tabular}{|c|c|c|c|c|c|c|c|c|}
\hline & \multicolumn{2}{|c|}{ LR } & \multicolumn{2}{|c|}{ XT } & \multicolumn{2}{|c|}{$\mathrm{CR}$} & \multicolumn{2}{|c|}{ GCNR } \\
\hline & Best & Worst & Best & Worst & Best & Worst & Best & Worst \\
\hline SLT & $\begin{array}{c}\text { SL-FDMAS } \\
(M L=10)\end{array}$ & DAS-T & - & - & $p$-DAS $(p=4)$ & DAS-T & $\operatorname{GCF}\left(M_{0}=3\right)$ & $\mathrm{SCF}(q=1)$ \\
\hline 4-MLT & $\begin{array}{c}\text { SL-FDMAS } \\
(M L=10)\end{array}$ & DAS-T & $p$-DAS $(p=4)$ & $\operatorname{PCF}(\gamma=0.2)$ & $p$-DAS $(p=4)$ & DAS-T & $p$-DAS $(p=3)$ & $\operatorname{SCF}(q=1)$ \\
\hline 6-MLT & $\begin{array}{c}\text { SL-FDMAS } \\
(M L=10)\end{array}$ & DAS-T & $p$-DAS $(p=4)$ & $\mathrm{PCF}(\gamma=0.2)$ & $p$-DAS $(p=4)$ & DAS-T & $p$-DAS $(p=3)$ & $\operatorname{SCF}(q=1)$ \\
\hline 8-MLT & $\begin{array}{c}\text { SL-FDMAS } \\
(M L=10)\end{array}$ & DAS-T & $p$-DAS $(p=4)$ & $\operatorname{PCF}(\gamma=0.2)$ & $p$-DAS $(p=4)$ & DAS-T & $G C F\left(M_{0}=2\right)$ & $\operatorname{SCF}(q=1)$ \\
\hline
\end{tabular}


beamformers can provide significant advantages in MLT as compared to DAS-T for all the analyzed parameters. Such improvements however are more or less significant depending on the design parameters of the algorithms, which can be properly tuned to achieve the best trade-off between image quality and frame rate. Particularly, the following performancemetrics trends can be highlighted (Fig. 3-4):

- increasing $M L$ in SL-FDMAS allows improving XT rejection, together with CR and GCNR, but worsens LR, as demonstrated in [27];

- $\quad$ with $p$-DAS, a higher $p$ value should be set to improve LR, XT rejection, and CR, unfortunately slightly reducing GCNR;

- $\quad$ LR does not significantly change when varying $M_{0}$ with GCF; XT and CR instead get worse for increasing values of $M_{0}$. The GCNR improves from CF to GCF with $M_{0}=1$, but then shows an almost constant trend for increasing $M_{0}$ values; however, the relative improvement with respect to DAS-T decreases on average with $M_{0}$;

- $\quad$ increasing $\gamma$ in PCF generally yields to an improvement of all image quality parameters;

- LR, XT and CR improve when increasing $q$ in SCF, while an opposite trend can be observed for GCNR; SCF in fact tends to degrade speckle uniformity (i.e. speckle has a high variance), so CNR worsens independently from possible dynamic range alterations;

- in all configurations (SLT/MLTs), the algorithms obtaining the worst performance parameters are the same (Table II): DAS-T for LR and CR, PCF with $\gamma=0.2$ for $\mathrm{XT}$ rejection, and $\mathrm{SCF}$ with $q=1$ for GCNR;

- the best LR is always achieved by SL-FDMAS with $M L=10$, while the best XT suppression and CR are always achieved by $p$-DAS with $p=4$, both in SLT and MLTs;

- $\quad$ in SLT and 8-MLT the best GCNR is that of GCF with $M_{0}=3$ and $M_{0}=2$, respectively, while in 4- and 6-MLT $p$ DAS (with $p=3$ ) achieves the best result.

Overall, we can say that $p$-DAS is probably the algorithm which allows achieving the best image quality in MLT, as it often yields to the highest performance metrics (Table II). In those few cases where it doesn't perform the best, it still reaches a good outcome: LR is only $\sim 0.1 \mathrm{~mm}$ lower than the best one achieved by SL-FDMAS with $M L=10$ and GCNR is only $\sim 0.01$ and 0.03 lower than that of GCF with $M_{0}=3$ and $M_{0}=2$ in SLT and 8-MLT, respectively.

Concerning the selection of the best MLT configuration, it should be based on the target imaging application, since a tradeoff exists between image quality and frame rate. In our case, we should also consider that with the 64-channel ULA-OP system higher MLTs imply lower signal-to-noise ratios (SNR), due to the reduction of TX signal amplitude proportionally to $N_{T X}$, as explained in Section II.G; thus, with the higher MLTs (e.g. 16MLT), image quality would probably be too low. However, in another work [1], exploiting the novel ULA-OP 256 research scanner, it was shown that the use of a virtual source for overlapping beams partially overcomes the reduction of SNR.

Finally, from a computational point of view, most of the analyzed methods could be efficiently implemented in real- time. Indeed, the computational complexity is $O(N)$ for $\mathrm{CF}$, PCF, SCF and $p$-DAS, while, for GCF, it is $O(N \cdot \log (N))$, since the Fast Fourier Transform (FFT) algorithm can be used. On the other hand, SL-FDMAS has a higher complexity, i.e. $O\left(N^{2}\right)$, due to signal cross-multiplications. However, in the case of standard FDMAS beamforming $(M L=63)$, it decreases again to $O(N)$, as the simplified formulation proposed in our previous paper [40] can be used. It is also worth highlighting that, in a real-time implementation, all the most complex operations ( $n$ th power, $n$-th root, phase calculation, etc.) would be implemented by means of look-up tables (LUT), as already shown in [40]. Such solution would make all the methods very efficient from a computational point of view.

One last comment should be done on the choice of techniques included in this work. Actually, we are aware that Short Lag Spatial Coherence (SLSC) [41], [42] should be considered among the main ultrasound imaging methods relying on the spatial coherence of backscattered signals. However, we preferred not to include it in this work, since our aim was to compare beamforming techniques for B-mode imaging only. SLSC instead provides images of the spatial coherence itself, and as such its outcome is not fairly and directly comparable to the considered B-mode imaging beamformers. For this reason, we are indeed investigating the behavior of this imaging modality with multiple beam transmission in a separate ongoing work. Moreover, it is worth highlighting that other techniques have been described in the literature to improve acoustic crosstalk suppression in MLT; they include e.g. the use of adaptive weights [19], the application of minimum variance adaptive beamforming in RX [22], orthogonal frequency division multiplexing [43], coded excitations [44] and harmonic imaging [45], but the comparison with those methods was out of the scope of this work, since they are not directly linked to the spatial coherence of backscattered signals.

\section{ACKNOWLEDGMENT}

The authors would like to thank Dr. G. Fradella (Careggi University Hospital, Florence, Italy) for his valuable contribution in performing in vivo acquisitions.

\section{REFERENCES}

[1] A. Ramalli et al., "Real-Time High-Frame-Rate Cardiac B-Mode and Tissue Doppler Imaging Based on Multiline Transmission and Multiline Acquisition," IEEE Trans. Ultrason., Ferroelectr., Freq. Control, vol. 65, no. 11, pp. 2030-2041, 2018.

[2] M. Cikes, L. Tong, G. R. Sutherland, and J. D'hooge, "Ultrafast cardiac ultrasound imaging: technical principles, applications, and clinical benefits," JACC Cardiovasc. Imaging, vol. 7, no. 8, pp. 812-823, 2014.

[3] S. Fadnes, M. S. Wigen, S. A. Nyrnes, and L. Lovstakken, "In Vivo Intracardiac Vector Flow Imaging Using Phased Array Transducers for Pediatric Cardiology," IEEE Transactions on Ultrasonics, Ferroelectrics, and Frequency Control, vol. 64, no. 9, pp. 1318-1326, Sep. 2017.

[4] P. Santos et al., "Natural shear wave imaging in the human heart: normal values, feasibility and reproducibility," IEEE Transactions on Ultrasonics, Ferroelectrics, and Frequency Control, pp. 1-1, 2018.

[5] A. Petrescu et al., "Velocities of Naturally Occurring Myocardial Shear Waves Increase With Age and in Cardiac Amyloidosis," JACC: Cardiovascular Imaging, p. 2908, Feb. 2019.

[6] J. Faurie, M. Baudet, J. Porée, G. Cloutier, F. Tournoux, and D. Garcia, "Coupling myocardium and vortex dynamics in diverging-wave 
echocardiography," IEEE Transactions on Ultrasonics, Ferroelectrics, and Frequency Control, pp. 1-1, 2018.

[7] P. Joos et al., "High-Frame-Rate Speckle-Tracking Echocardiography," IEEE Transactions on Ultrasonics, Ferroelectrics, and Frequency Control, vol. 65, no. 5, pp. 720-728, May 2018.

[8] J. Grondin, V. Sayseng, and E. E. Konofagou, "Cardiac Strain Imaging with Coherent Compounding of Diverging Waves," IEEE Trans. Ultrason., Ferroelectr., Freq. Control, vol. 64, no. 8, pp. 1212-1222, 2017.

[9] L. Demi, "Practical Guide to Ultrasound Beam Forming: Beam Pattern and Image Reconstruction Analysis," Applied Sciences, vol. 8, no. 9, p. 1544, Sep. 2018.

[10] C. Papadacci, M. Pernot, M. Couade, M. Fink, and M. Tanter, "Highcontrast ultrafast imaging of the heart," IEEE Trans. Ultrason., Ferroelectr., Freq. Control, vol. 61, no. 2, pp. 288-301, 2014.

[11] H. Hasegawa and H. Kanai, "High-frame-rate echocardiography using diverging transmit beams and parallel receive beamforming," J. Med. Ultrasonics, vol. 38, no. 3, pp. 129-140, Jul. 2011.

[12] R. Mallart and M. Fink, "Improved imaging rate through simultaneous transmission of several ultrasound beams," in New Developments in Ultrasonic Transducers and Transducer Systems, 1992, vol. 1733, pp. $120-130$.

[13] L. Tong, A. Ramalli, R. Jasaityte, P. Tortoli, and J. D'hooge, "Multitransmit beam forming for fast cardiac imaging--experimental validation and in vivo application," IEEE Trans. Med. Imaging, vol. 33, no. 6, pp. 1205-1219, 2014.

[14] D. P. Shattuck, M. D. Weinshenker, S. W. Smith, and O. T. von Ramm, "Explososcan: a parallel processing technique for high speed ultrasound imaging with linear phased arrays," J. Acoust. Soc. Am., vol. 75, no. 4, pp. 1273-1282, 1984.

[15] O. T. von Ramm, S. W. Smith, and H. G. Pavy, "High-speed ultrasound volumetric imaging system. II. Parallel processing and image display," IEEE Trans. Ultrason., Ferroelectr., Freq. Control, vol. 38, no. 2, pp. 109-115, Mar. 1991.

[16] G. Montaldo, M. Tanter, J. Bercoff, N. Benech, and M. Fink, "Coherent plane-wave compounding for very high frame rate ultrasonography and transient elastography," IEEE Trans. Ultrason., Ferroelectr., Freq. Control, vol. 56, no. 3, pp. 489-506, 2009.

[17] G. Matrone, A. S. Savoia, G. Caliano, and G. Magenes, "Ultrasound plane-wave imaging with delay multiply and sum beamforming and coherent compounding," in 201638 th Annual International Conference of the IEEE Engineering in Medicine and Biology Society (EMBC), 2016, pp. 3223-3226.

[18] L. Tong, H. Gao, and J. D'hooge, "Multi-transmit beam forming for fast cardiac imaging--a simulation study," IEEE Trans. Ultrason., Ferroelectr., Freq. Control, vol. 60, no. 8, pp. 1719-1731, Aug. 2013.

[19] G. Zurakhov et al., "Multiline Transmit Beamforming Combined With Adaptive Apodization," IEEE Trans. Ultrason., Ferroelectr., Freq. Control, vol. 65, no. 4, pp. 535-545, Apr. 2018.

[20] A. Rodriguez-Molares, H. Torp, B. Denarie, and L. Løvstakken, "The angular apodization in coherent plane-wave compounding [Correspondence]," IEEE Trans. Ultrason., Ferroelectr., Freq. Control, vol. 62, no. 11, pp. 2018-2023, Nov. 2015.

[21] G. Matrone, A. Ramalli, A. S. Savoia, P. Tortoli, and G. Magenes, "High Frame-Rate, High Resolution Ultrasound Imaging With MultiLine Transmission and Filtered-Delay Multiply And Sum Beamforming," IEEE Trans. Med. Imaging, vol. 36, no. 2, pp. 478-486, Feb. 2017.

[22] A. Rabinovich, A. Feuer, and Z. Friedman, "Multi-line transmission combined with minimum variance beamforming in medical ultrasound imaging," IEEE Trans. Ultrason., Ferroelectr., Freq. Control, vol. 62, no. 5, pp. 814-827, May 2015.

[23] W. Guo, Y. Wang, and J. Yu, "A Sibelobe Suppressing Beamformer for Coherent Plane Wave Compounding," Appl.Sci., vol. 6, no. 11, p. 359, Nov. 2016.

[24] I. K. Holfort, F. Gran, and J. A. Jensen, "Plane wave medical ultrasound imaging using adaptive beamforming," in Proceedings of the 5th IEEE Sensor Array and Multichannel Signal Processing Workshop, 2008, pp. 288-292.

[25] B. Lokesh and A. K. Thittai, "Spatial resolution improvement in plane wave imaging using adaptive sign coherence factor weighting," in 2016 IEEE Ultrasonics Symposium, Tours, France, 2016.

[26] J. Zhao, Y. Wang, X. Zeng, J. Yu, B. Y. S. Yiu, and A. C. H. Yu, "Plane wave compounding based on a joint transmitting-receiving adaptive beamforming," IEEE Trans. Ultrason., Ferroelectr., Freq. Control, vol. 62 , no. 8, pp. 1440-1452, 2015.

[27] G. Matrone and A. Ramalli, "Spatial Coherence of Backscattered Signals in Multi-Line Transmit Ultrasound Imaging and Its Effect on Short-Lag Filtered-Delay Multiply and Sum Beamforming," Applied Sciences, vol. 8, no. 4, p. 486, Apr. 2018.

[28] P.-C. Li and M.-L. Li, "Adaptive imaging using the generalized coherence factor," IEEE Trans. Ultrason., Ferroelectr., Freq. Control, vol. 50, no. 2, pp. 128-142, 2003.

[29] G. Matrone, A. S. Savoia, G. Caliano, and G. Magenes, "The Delay Multiply and Sum Beamforming Algorithm in Ultrasound B-Mode Medical Imaging," IEEE Trans. Med. Imaging, vol. 34, no. 4, pp. 940949,2015

[30] M. Polichetti, F. Varray, J.-C. Béra, C. Cachard, and B. Nicolas, "A Nonlinear Beamformer Based on p-th Root Compression-Application to Plane Wave Ultrasound Imaging," Appl. Sci., vol. 8, no. 4, p. 599, 2018.

[31] J. Camacho, M. Parrilla, and C. Fritsch, "Phase coherence imaging," IEEE Trans. Ultrason., Ferroelectr., Freq. Control, vol. 56, no. 5, pp. 958-974, 2009.

[32] R. Mallart and M. Fink, "Adaptive focusing in scattering media through sound-speed inhomogeneities: The van Cittert Zernike approach and focusing criterion," J. Acoust. Soc. Am., vol. 96, no. 6, pp. 3721-3732, 1994.

[33] K. W. Hollman, K. W. Rigby, and M. O'Donnell, "Coherence factor of speckle from a multi-row probe," in 1999 IEEE Ultrasonics Symposium, 1999, vol. 2, pp. 1257-1260 vol.2.

[34] M. Polichetti et al., "A Computationally Efficient Non-Linear Beamformer Based on pth Root Signal Compression for Enhanced Ultrasound B-Mode Imaging," in 2017 IEEE International Ultrasonics Symposium (IUS), Washington DC, USA, 2017.

[35] E. Boni et al., "A reconfigurable and programmable FPGA-based system for nonstandard ultrasound methods," IEEE Trans. Ultrason., Ferroelectr., Freq. Control, vol. 59, no. 7, pp. 1378-1385, Jul. 2012.

[36] E. Boni, A. Cellai, A. Ramalli, and P. Tortoli, "A high performance board for acquisition of 64-channel ultrasound RF data," in 2012 IEEE International Ultrasonics Symposium, 2012, pp. 2067-2070.

[37] A. Ramalli, L. Tong, J. Luo, J. d'Hooge, and P. Tortoli, "Safety of fast cardiac imaging using multiple transmit beams: Experimental verification," in 2014 IEEE Ultrasonics Symposium, Chicago, IL, USA, 2014.

[38] A. Rodriguez-Molares, O. M. H. Rindal, J. D’hooge, S. Måsøy, A. Austeng, and H. Torp, "The Generalized Contrast-to-Noise Ratio," in 2018 IEEE International Ultrasonics Symposium (IUS), 2018, pp. 1-4.

[39] O. M. H. Rindal, A. Austeng, A. Fatemi, and A. Rodriguez-Molares, "The effect of dynamic range alterations in the estimation of contrast," IEEE Transactions on Ultrasonics, Ferroelectrics, and Frequency Control, pp. 1-1, 2019.

[40] A. Ramalli et al., "High dynamic range ultrasound imaging with realtime filtered-delay multiply and sum beamforming," in 2017 IEEE International Ultrasonics Symposium (IUS), 2017, pp. 1-4.

[41] M. A. Lediju, G. E. Trahey, B. C. Byram, and J. J. Dahl, "Short-lag spatial coherence of backscattered echoes: imaging characteristics," IEEE Transactions on Ultrasonics, Ferroelectrics, and Frequency Control, vol. 58, no. 7, pp. 1377-1388, Jul. 2011.

[42] M. A. Lediju Bell, R. Goswami, J. A. Kisslo, J. J. Dahl, and G. E. Trahey, "Short-Lag Spatial Coherence (SLSC) Imaging of Cardiac Ultrasound Data: Initial Clinical Results," Ultrasound Med Biol, vol. 39, no. 10, pp. 1861-1874, Oct. 2013.

[43] L. Demi, A. Ramalli, E. Boni, and J. D’hooge, "Orthogonal Frequency Division Multiplexing Combined with Multi Line Transmission for Ultrafast Ultrasound Imaging: Experimental Findings," in 2018 IEEE International Ultrasonics Symposium (IUS), 2018, pp. 1-4.

[44] L. Tong et al., "Coded Excitation for Crosstalk Suppression in Multiline Transmit Beamforming: Simulation Study and Experimental Validation," Applied Sciences, vol. 9, no. 3, p. 486, Jan. 2019.

[45] F. Prieur, B. Dénarié, A. Austeng, and H. Torp, "Multi-line transmission in medical imaging using the second-harmonic signal," IEEE Trans. Ultrason., Ferroelectr., Freq. Control, vol. 60, no. 12, pp. 2682-2692, Dec. 2013. 